\title{
Prediction-Correction Alternating Direction Method for Power Systems Economic Dispatch
}

\author{
Yaming Ren*, Shumin Fei, Haikun Wei \\ Department of Automation, Southeast University, Nanjing, 210096, China. \\ * Corresponding author. Tel.: 86-25-8379-2719; email: renyaming1981@gmail.com \\ Manuscript submitted November 3, 2014; accepted April 20, 2015. \\ doi: 10.17706/ijcee.2015.7.3.179-188
}

\begin{abstract}
The alternating direction method is a powerful tool for solving multi-area economic dispatch problem. However, plenty of applications show that the choice of step size and penalty parameter has an important influence on algorithm's convergence rate. In this paper, we proposed a novel prediction-correction algorithm. To be more exact, the prediction is generated by original alternating direction method, while the correction is implemented via adjusting step size and penalty parameter. Numerical results illustrate the proposed method is robust.
\end{abstract}

Key words: Multi-area economic dispatch, augmented lagrangian relaxation, alternating direction method.

\section{Introduction}

With the growing scale of power systems, ref. [1] provides a general framework for multi-area economic dispatch (MADE) problem with tie-line constraints. The conventional and traditional centralized economic dispatch algorithms suffer from dimensionality problem and bottleneck of data transmission. As a result, it is critical to propose a more effective strategy to cope with MADE problem.

Many literatures with regard to distributed computation attempted to solve multi-area economic dispatch problem. In [2], the decomposition-coordination interior point method (DCIPM) is applied to solve multi-area optimal reactive power flow problem. However, DCIPM requires that each area must use interior point method, this is difficult to achieve in practice. Particle swarm optimization (PSO) is introduced to eliminate the MAED problem [3]. The positive aspect of PSO is that it only depends on the objective function information, i.e. the objective function does not require some special properties, for example, smoothness and convexity etc. However, the negative aspect of PSO is that the transmission bottleneck is still in existence.

Traditional alternating direction method (ADM) is employed to solve generation scheduling problem in interconnected power systems [4]. However, the convergence rate of ADM is sensitive to the selection of penalty parameter and step size. In this paper, a novel prediction-correction alternating direction method (PCADM) is derived from the traditional ADM. To be more exact, the prediction is generated by ADM, and the correction is to achieve proper step size and penalty parameter based on iterative information.

\section{Formulation of Multi-area Economic Dispatch}

The objective of multi-area economic dispatch is to minimize the total active power production cost [5], [6]. In fact, multi-area economic dispatch problem can be described as convex programming problem with 
linear constraints between different areas. A mathematical formulation of two-area economic dispatch problem is expressed as follows:

$$
\begin{aligned}
& \min F=f\left(x_{1}\right)+g\left(x_{2}\right) \\
& \text { Subject to }: x_{1} \in \Omega_{1}, x_{2} \in \Omega_{2} \\
& \qquad \Theta\left(x_{1}, x_{2}\right) \equiv A x_{1}+B x_{2}=0
\end{aligned}
$$

where $f: R^{n} \rightarrow R$ and $g: R^{m} \rightarrow R$ are convex function, and present the total fuel cost in area 1 and area 2 , respectively. $\Omega_{1} \in R^{n}$ and $\Omega_{2} \in R^{m}$ are given closed sets while satisfying the corresponding constraints, such as power balance and limits on variables. $\Theta\left(x_{1}, x_{2}\right)$ is the linear consistency constraint between two areas, during which $A \in R^{r \times n}$ and $B \in R^{r \times m}$ are given matrices.

We employ standard lagrangian relaxation method to deal with problem (1). That is to attach a lagrangian multiplier vector $\lambda \in R^{r}$ to the linear consistency constraint $\Theta\left(x_{1}, x_{2}\right)$, the reformulation of problem (1) is expressed as follow:

$$
\begin{aligned}
& \min F=f\left(x_{1}\right)+g\left(x_{2}\right)-\lambda^{T}\left(A x_{1}+B x_{2}\right) \\
& \text { Subject to : } x_{1} \in \Omega_{1}, x_{2} \in \Omega_{2}
\end{aligned}
$$

The optimization problem (2) is equivalent to solving a saddle-point problem as shown in (3) and (4), [7]. To be exact, the new iteration $\left(x_{1}^{k+1}, x_{2}^{k+1}, \lambda^{k+1}\right)$ is generated from given vector $\left(x_{1}^{k}, x_{2}^{k}, \lambda^{k}\right)$ by the following scheme:

$\left(x_{1}^{k+1}, x_{2}^{k+1}\right)$ is obtained by solving

$$
\left(x_{1}^{k+1}, x_{2}^{k+1}\right)=\min \left\{f\left(x_{1}\right)+g\left(x_{2}\right)-\lambda^{T, k}\left(A x_{1}+B x_{2}\right) \mid x_{1} \in \Omega_{1}, x_{2} \in \Omega_{2}\right\}
$$

Then, $\lambda^{k+1}$ is obtained by updating

$$
\lambda^{k+1}=\lambda^{k}-c\left(A x_{1}^{k+1}+B x_{2}^{k+1}\right)
$$

One main advantage of (3) is that the objective function is separable when lagrangian multiplier vector is held fixed. However, it is difficult to ensure the convex property of objective function. In fact, solving non-convex programming problem is difficult compared with convex programming problem. Consequently, augmented lagrangian relaxation method (ALR) is employed to cope with equality constraint $\Theta\left(x_{1}, x_{2}\right)$, and then problem (1) can been rewritten as follows:

$$
\begin{aligned}
& \min \left\{f\left(x_{1}\right)+g\left(x_{2}\right)-\lambda^{T}\left(A x_{1}+B x_{2}\right)+\frac{c}{2}\left\|A x_{1}+B x_{2}\right\|^{2}\right\} \\
& \text { Subject to : } x_{1} \in \Omega_{1}, x_{2} \in \Omega_{2}
\end{aligned}
$$

where $c>0$ is given constant and is a penalty factor for linear constraint, the Euclidean norm of vector 
$x$ can be denoted by $\|x\|$, i.e., $\|x\|=\sqrt{x^{T} x}$.

Similarly, the optimization problem (5) is equivalent to solving a saddle-point problem via the following iterative scheme:

$$
\left(x_{1}^{k+1}, x_{2}^{k+1}\right)=\min \left\{f\left(x_{1}\right)+g\left(x_{2}\right)-\lambda^{T, k}\left(A x_{1}+B x_{2}\right)+\frac{c}{2}\left\|A x_{1}+B x_{2}\right\|^{2} \mid x_{1} \in \Omega_{1}, x_{2} \in \Omega_{2}\right\}
$$

Then, $\lambda^{k+1}$ is obtained by updating

$$
\lambda^{k+1}=\lambda^{k}-c\left(A x_{1}^{k+1}+B x_{2}^{k+1}\right)
$$

It is clear that the sum of convex functions is still convex function. We have known that $f\left(x_{1}\right)$ and $g\left(x_{2}\right)$ are convex. In addition, if we view $\left(A x_{1}+B x_{2}\right)$ as a whole, then we can find that $-\lambda^{T}\left(A x_{1}+B x_{2}\right)+\frac{c}{2}\left\|A x_{1}+B x_{2}\right\|^{2}$ is quadratic function. As a result, we know the objective function of problem (5) generated by ALR is convex. Using augmented lagrangian relaxation method to deal with equal constraint ensures the convex property of objective function.

\section{Solution}

\subsection{Alternating Direction Method (ADM)}

The alternating directions method (ADM) is a powerful method for solving (6) [8], [9]. Taking advantage of ADM, problem (5) can be solved with the following iterative scheme.

Step 1: Compute:

$$
x_{1}^{k+1}=\arg \min \left\{f\left(x_{1}\right)+\frac{c}{2}\left\|A x_{1}+B x_{2}^{k}\right\|^{2}-\lambda^{T, k} A x_{1}\right\}
$$

Step 2: Compute:

$$
x_{2}^{k+1}=\arg \min \left\{g\left(x_{2}\right)+\frac{c}{2}\left\|A x_{1}^{k+1}+B x_{2}\right\|^{2}-\lambda^{T, k} B x_{2}\right\}
$$

Step 3: Lagrange multiplier updating:

$$
\lambda^{k+1}=\lambda^{k}-c\left(A x_{1}^{k+1}+B x_{2}^{k+1}\right)
$$

Step 4: Check the stop criterion. If $\max \left(\left\|\lambda^{k+1}-\lambda^{k}\right\|,\left\|B x_{2}^{k}-B x_{2}^{k+1}\right\|\right)<\eta$ then stop. If not $k=k+1$, go to Step 1 .

\subsection{Prediction-Correction Alternating Direction Method (PCADM)}

In this section, we propose a prediction-correction alternating direction method (PCADM), which is 
derived from the classical ADM. To be more precise, the prediction is generated by ADM, and the correction is to adjust step size and penalty parameter.

\subsubsection{Adjusting step size}

For convenience, we denote

$$
N=\left(\begin{array}{ll}
c B^{T} B & 0 \\
0 & \frac{1}{c} I_{n_{b}}
\end{array}\right), w=\left(\begin{array}{c}
x_{1} \\
x_{2} \\
\lambda
\end{array}\right), v=\left(\begin{array}{c}
x_{2} \\
\lambda
\end{array}\right)
$$

LEMMA 1

$$
\left(\tilde{v}^{k}-v^{*}\right)^{T} N\left(v^{k}-\tilde{v}^{k}\right)-\left(\lambda^{k}-\tilde{\lambda}^{k}\right)^{T} B\left(x_{2}^{k}-\tilde{x}_{2}^{k}\right) \geq 0
$$

Proof: Let $\tilde{w}^{k} \in W$ is generated by the iterative scheme of ADM from given $w^{k}$, we get [10],

$$
\begin{aligned}
& f\left(x_{1}\right)+g\left(x_{2}\right)-f\left(\tilde{x}_{1}^{k}\right)-g\left(\tilde{x}_{2}^{k}\right)+\left(w-\tilde{w}^{k}\right)^{T} \\
& \left\{\left(\begin{array}{l}
-A^{T} \tilde{\lambda}^{k}+c A^{T}\left(A \tilde{x}_{1}^{k}+B \tilde{x}_{2}^{k}\right) \\
-B^{T} \tilde{\lambda}^{k}+c B^{T}\left(A \tilde{x}_{1}^{k}+B \tilde{x}_{2}^{k}\right) \\
\left(A \tilde{x}_{1}^{k}+B \tilde{x}_{2}^{k}\right)
\end{array}\right)+\left(\begin{array}{lll}
0 & -c A^{T} B & A^{T} \\
0 & 0 & B^{T} \\
0 & 0 & \frac{1}{c} I_{m}
\end{array}\right)\left(\tilde{w}^{k}-w^{k}\right)\right\} \geq 0
\end{aligned}
$$

Setting $w=w^{*}$ in (13), we get,

$$
\left(w^{*}-\tilde{w}^{k}\right)^{T}\left(\begin{array}{lll}
0 & -c A^{T} B & 0 \\
0 & 0 & 0 \\
0 & 0 & \frac{1}{c} I
\end{array}\right)\left(\tilde{w}^{k}-w^{k}\right) \geq f\left(\tilde{x}_{1}^{k}\right)+g\left(\tilde{x}_{2}^{k}\right)-f\left(x_{1}^{*}\right)-g\left(x_{2}^{*}\right)+\left(\tilde{w}^{k}-w^{*}\right)^{T} F\left(\tilde{w}^{k}\right)
$$

where $F(w)=\left(-A^{T} \lambda,-B^{T} \lambda, A x_{1}-B x_{2}\right)^{T}$

According to [10], we get,

$$
\begin{gathered}
\left(\tilde{w}^{k}-w^{*}\right)^{T} F\left(\tilde{w}^{k}\right) \geq\left(\tilde{w}^{k}-w^{*}\right)^{T} F\left(w^{*}\right) \\
f\left(\tilde{x}_{1}^{k}\right)+g\left(\tilde{x}_{2}^{k}\right)-f\left(x_{1}^{*}\right)-g\left(x_{2}^{*}\right)+\left(\tilde{w}^{k}-w^{*}\right)^{T} F\left(w^{*}\right) \geq 0
\end{gathered}
$$

Combing (14)-(16), we get 


$$
\begin{aligned}
& \left(w^{*}-\tilde{w}^{k}\right)^{T}\left(\begin{array}{ccc}
0 & -c A^{T} B & 0 \\
0 & 0 & 0 \\
0 & 0 & \frac{1}{c} I
\end{array}\right)\left(\tilde{w}^{k}-w^{k}\right) \geq 0 \\
& \Rightarrow\left(w^{*}-\tilde{w}^{k}\right)^{T}\left(\begin{array}{lll}
0 & 0 & 0 \\
0 & c B^{T} B & 0 \\
0 & 0 & \frac{1}{c} I_{m}
\end{array}\right)\left(\tilde{w}^{k}-w^{k}\right)-\left(\lambda^{k}-\tilde{\lambda}^{k}\right)^{T} B\left(y^{k}-\tilde{y}^{k}\right) \geq 0 \\
& \Rightarrow\left(\tilde{v}^{k}-v^{*}\right)^{T} N\left(v^{k}-\tilde{v}^{k}\right)-\left(\lambda^{k}-\tilde{\lambda}^{k}\right)^{T} B\left(x_{2}^{k}-\tilde{x}_{2}^{k}\right) \geq 0 \\
& \Rightarrow\left(v^{k}-v^{*}\right)^{T} N\left(v^{k}-\tilde{v}^{k}\right) \geq\left(v^{k}-\tilde{v}^{k}\right)^{T} N\left(v^{k}-\tilde{v}^{k}\right)+\left(\lambda^{k}-\tilde{\lambda}^{k}\right)^{T} B\left(x_{2}^{k}-\tilde{x}_{2}^{k}\right)
\end{aligned}
$$

According to (17) and using

$$
\begin{aligned}
& \left(v^{k}-\tilde{v}^{k}\right)^{T} N\left(v^{k}-\tilde{v}^{k}\right)+\left(\lambda^{k}-\tilde{\lambda}^{k}\right)^{T} B\left(x_{2}^{k}-\tilde{x}_{2}^{k}\right) \\
= & \frac{1}{2}\left(v^{k}-\tilde{v}^{k}\right)^{T}\left(\begin{array}{ll}
c B^{T} B & 0 \\
0 & \frac{1}{c} I_{n_{b}}
\end{array}\right)\left(v^{k}-\tilde{v}^{k}\right)+\frac{1}{2}\left(v^{k}-\tilde{v}^{k}\right)^{T}\left(\begin{array}{ll}
c B^{T} B & 2 B \\
0 & \frac{1}{c} I_{n_{b}}
\end{array}\right)\left(v^{k}-\tilde{v}^{k}\right)
\end{aligned}
$$

We get

$$
\left(v^{k}-\tilde{v}^{k}\right)^{T} N\left(v^{k}-\tilde{v}^{k}\right)+\left(\lambda^{k}-\tilde{\lambda}^{k}\right)^{T} B\left(x_{2}^{k}-\tilde{x}_{2}^{k}\right) \geq 0
$$

Based on above discussion, the proof of Lemma 1 is completed.

LEMMA 2

$$
\left\|v^{k}-v^{*}\right\|_{N}^{2}-\left\|v^{k+1}-v^{*}\right\|_{N}^{2} \geq\left\|v^{k}-v^{k+1}\right\|_{N}^{2}
$$

Proof: using the $\tilde{v}^{k}$ produced by scheme of ADM, update the new iterate $v^{k+1}$ by

$$
v^{k+1}=v^{k}-\alpha\left(v^{k}-\tilde{v}^{k}\right)
$$

where $\alpha$ is the step size.

By using LEMMA 1 and (21), we obtain

$$
\begin{aligned}
\varphi(\alpha) & =\left\|v^{k}-v^{*}\right\|_{N}^{2}-\left\|v^{k+1}-v^{*}\right\|_{N}^{2} \\
& =\left\|v^{k}-v^{*}\right\|_{N}^{2}-\left\|v^{k}-v^{*}-\alpha\left(v^{k}-\tilde{v}^{k}\right)\right\|_{N}^{2} \\
& =2 \alpha\left\langle v^{k}-v^{*}, N\left(v^{k}-\tilde{v}^{k}\right)\right\rangle-\alpha^{2}\left\|\left(v^{k}-\tilde{v}^{k}\right)\right\|_{N}^{2} \\
& \geq 2 \alpha\left(\left(v^{k}-\tilde{v}^{k}\right)^{T} N\left(v^{k}-\tilde{v}^{k}\right)+\left(\lambda^{k}-\tilde{\lambda}^{k}\right)^{T} B\left(x_{2}^{k}-\tilde{x}_{2}^{k}\right)\right)-\alpha^{2}\left\|v^{k}-\tilde{v}^{k}\right\|_{N}^{2}
\end{aligned}
$$


In fact, the quadratic function $q(\alpha)$ with lower bound value of $\varphi(\alpha)$ is obtained via (22),

$$
q(\alpha)=2 \alpha\left(\left(v^{k}-\tilde{v}^{k}\right)^{T} N\left(v^{k}-\tilde{v}^{k}\right)+\left(\lambda^{k}-\tilde{\lambda}^{k}\right)^{T} B\left(x_{2}^{k}-\tilde{x}_{2}^{k}\right)\right)-\alpha^{2}\left\|v^{k}-\tilde{v}^{k}\right\|_{N}^{2}
$$

$q(\alpha)$ is a quadratic function of $\alpha$ and it reaches its maximum at

$$
\alpha_{k}=\frac{\left\|v^{k}-\tilde{v}^{k}\right\|_{N}^{2}+\left(\lambda^{k}-\tilde{\lambda}^{k}\right)^{T} B\left(x_{2}^{k}-\tilde{x}_{2}^{k}\right)}{\left\|v^{k}-\tilde{v}^{k}\right\|_{N}^{2}}
$$

Therefore, we choose $\alpha^{k}$ as the optimal step size.

Combing (23) and (24), we get

$$
\begin{aligned}
q\left(\alpha_{k}\right) & =2 \alpha_{k}\left(\left(v^{k}-\tilde{v}^{k}\right)^{T} N\left(v^{k}-\tilde{v}^{k}\right)+\left(\lambda^{k}-\tilde{\lambda}^{k}\right)^{T} B\left(x_{2}^{k}-\tilde{x}_{2}^{k}\right)\right)-\left(\alpha_{k}\right)^{2}\left\|v^{k}-\tilde{v}^{k}\right\|_{N}^{2} \\
& =\left(\alpha_{k}\right)^{2}\left\|v^{k}-\tilde{v}^{k}\right\|_{N}^{2} \\
& =\left\|v^{k}-v^{k+1}\right\|_{N}^{2} \geq 0
\end{aligned}
$$

Based on above discussion, the proof of Lemma 2 is completed.

Then, we can say that the sequence $\left\{v^{k}\right\}$ leads that [11],

$$
\lim _{k \rightarrow \infty} \lambda^{k}=\lambda^{*}, \lim _{k \rightarrow \infty} B x_{2}^{k}=B x_{2}^{*}
$$

Interestingly, (26) is consistent with ADM's stop criterion, so the strategy of adjusting step size can be written as follows.

$$
v^{k+1}=v^{k}-\alpha_{k}\left(v^{k}-\tilde{v}^{k}\right)
$$

\subsubsection{Adjusting penalty parameter}

In fact, a great number of experiments have illustrated that the choice of penalty factors has significant influence on convergence rate of ADM, so it is important to propose a strategy about how to adjust penalty parameter.

According to [12], solving problem (3) is equivalent to finding a point which satisfies $e(w)=0$.

$$
e(w)=\left[\begin{array}{l}
e_{x_{1}}(w) \\
e_{x_{2}}(w) \\
e_{\lambda}(w)
\end{array}\right]=\left[\begin{array}{l}
x_{1}-P_{\Omega 1}\left\{x_{1}-\left[\nabla f\left(x_{1}\right)-A^{T} \lambda\right]\right\} \\
x_{2}-P_{\Omega 2}\left\{x_{2}-\left[\nabla g\left(x_{2}\right)-B^{T} \lambda\right]\right\} \\
A x_{1}-B x_{2}
\end{array}\right]
$$

where $P_{\Omega}(\bullet)$ denotes the projection on $\Omega$. $\nabla f(\bullet)$ denotes the gradient of $f(\bullet)$. 
Fortunately, it can be proved that $e_{x_{2}}(w)=0$. As a result, for the sake of balance, we just need to ensure $e_{x_{2}}(w) \approx e_{\lambda}(w)$. It is to say that if $e_{x_{2}}(w) \leq e_{\lambda}(w)$, we should increase penalty factor in the next iteration; by contract, if $e_{x_{2}}(w) \geq e_{\lambda}(w)$, we should increase penalty factor in the next iteration. Three different strategies related to how to adjust penalty parameter has been presented, more details about these strategies can be found in [12].

Strategy 1: penalty parameter is monotonically increasing,

$$
c= \begin{cases}c \times 2 & \text { if }\left\|e_{x_{1}}\left(w^{k}\right)\right\|<0.1 \times\left\|e_{\lambda}\left(w^{k}\right)\right\| \\ c & \text { otherwise }\end{cases}
$$

Strategy 2: penalty parameter is monotonically decreasing

$$
c= \begin{cases}c \times 0.5 & \text { if } 0.1 \times\left\|e_{x_{1}}\left(w^{k}\right)\right\|>\left\|e_{\lambda}\left(w^{k}\right)\right\| \\ c & \text { otherwise }\end{cases}
$$

Strategy 3: penalty parameter is self-adaptive,

$$
c= \begin{cases}c \times 2 & \text { if }\left\|e_{x_{1}}\left(w^{k}\right)\right\|<0.1 \times\left\|e_{\lambda}\left(w^{k}\right)\right\| \\ c \times 0.5 & \text { if } 0.1 \times\left\|e_{x_{1}}\left(w^{k}\right)\right\|>\left\|e_{\lambda}\left(w^{k}\right)\right\| \\ c & \text { otherwise }\end{cases}
$$

If we employ strategy 1 , the penalty parameter sequence is increasing monotonically. As a result, the value of starting penalty parameter has to be significantly small in order to ensure the convergence of strategy 1 . Similarly, if we use strategy 2 , the penalty parameter sequence is decreasing monotonically. Consequently, the value of starting penalty parameter has to be markedly large ensuring the convergence of strategy 2. Based on the discussion above, it is clear that both strategies 1 and 2 are sensitive to the starting penalty parameter. However, it is always difficult to obtain a proper starting penalty parameter ahead of time. Comparing with strategies 1 and 2, we find that strategy 3 is more effective and flexible. Specially, the convergence performance of strategy 3 is independent of the starting penalty parameter. As a consequence, in this paper, we use strategy 3 to adjust penalty parameter.

Then, the self-adaptive penalty parameter alternating direction method (PCADM) can be expressed as follows.

Step 1: Compute

$$
x_{1}^{k+1}=\arg \min \left\{f\left(x_{1}\right)+\frac{c}{2}\left\|A x_{1}+B x_{2}^{k}\right\|^{2}-\lambda^{T, k} A x_{1}\right\}
$$

Step 2: Compute

$$
x_{2}^{k+1}=\arg \min \left\{g\left(x_{2}\right)+\frac{c}{2}\left\|A x_{1}^{k+1}+B x_{2}\right\|^{2}-\lambda^{T, k} B x_{2}\right\}
$$


Step 3: Lagrange multiplier updating

$$
\lambda^{k+1}=\lambda^{k}-c\left(A x_{1}^{k+1}+B x_{2}^{k+1}\right)
$$

Step 4: Check the stop criterion. If $\max \left(\left\|\lambda^{k+1}-\lambda^{k}\right\|,\left\|B x_{2}^{k}-B x_{2}^{k+1}\right\|\right)<\eta$ then stop. If not $k=k+1$. If $k>k_{\max }$, then stop ,the algorithm fails to converge with maximal iteration $k_{\max }$, if not, go to Step 5.

Step 5: Updating penalty parameter $c$, then go to Step 6.

$$
c= \begin{cases}c \times 2 & \text { if }\left\|e_{x_{1}}\left(w^{k}\right)\right\|<0.1 \times\left\|e_{\lambda}\left(w^{k}\right)\right\| \\ c \times 0.5 & \text { if } 0.1 \times\left\|e_{x_{1}}\left(w^{k}\right)\right\|>\left\|e_{\lambda}\left(w^{k}\right)\right\| \\ c & \text { otherwise }\end{cases}
$$

Step 6: Compute, then go to step 1.

$$
v^{k+1}=v^{k}-\alpha\left(v^{k}-\tilde{v}^{k}\right)
$$

\section{Computational Examples and Analysis}

\subsection{Test System: 4-Unit System}

In this section, a two-area 4-unit system is studied [13]. Area 1 has two unites and the demand is set to be 560MW. Similarly, area 2 has two unites and the demand is set to be $240 \mathrm{MW}$. The transmission capacity limit between area 1 and area 2 is set to be 200MW. Furthermore, network losses are neglected in this test system. Throughout this paper, the stop criterion and initial value of lagrangian multiplier are set to be $\eta=$ $10^{-4}, \lambda=0$.

\subsection{Comparative Analysis}

In order to demonstrate the feasibility and effectiveness of PCADM. The given 4-unit system is solved via using three different methods for the multi-area economic dispatch problem:

1) The classic alternating direction method (ADM);

2) The traditional auxiliary problem principle (APP) [14], [15];

3) The proposed self-adaptive penalty parameter alternating direction method (PCADM).

Table 1 shows corresponding numerical results. To be more accurate, the first and second columns indicate the method applied and different penalty factor $c$. After that the third column shows the total number of iteration reaching the optimum. The fourth column shows total active power output cost. As can be seen from Table 1 that the PCADM has better performance with different penalty factor $c$ by comparing the results obtained using standard ADM and APP. On the other hand, PCADM is robust in terms of the choice of penalty parameter $c$.

\section{Conclusions}

The objective of this paper is to solve multi-area security constrained economic dispatch problem in the form of the distributed computing efficiently. In this paper, the proposed PCADM is derived from the 
classical alternating direction method. More precisely, the focus of PCADM is to adjust step size and penalty factors according to iterative information. A 4-unit, two-area test systems has been employed to illustrate the correctness of proposed PCADM, For the purpose of comparisons, the results obtained via traditional ADM are considered, numerical results reveal the proposed PCADM has good adaptability to the selection of different penalty parameter.

Table 1. Comparison of Optimization Results

\begin{tabular}{lllc}
\hline \hline Method & Penalty factor & Iterations & Total cost $(\$ / \mathrm{h})$ \\
\hline & $5 \times 10^{0}$ & Fail to converge with maximal iteration 100 \\
& $5 \times 10^{-1}$ & Fail to converge with maximal iteration 100 \\
ADM & $5 \times 10^{-2}$ & Fail to converge with maximal iteration 100 \\
& $5 \times 10^{-3}$ & 27 & $7.7549 \times 10^{3}$ \\
& $5 \times 10^{-4}$ & Fail to converge with maximal iteration 100 \\
\hline \multirow{4}{*}{ APP } & $5 \times 10^{0}$ & Fail to converge with maximal iteration 100 \\
& $5 \times 10^{-1}$ & Fail to converge with maximal iteration 100 \\
& $5 \times 10^{-2}$ & 65 & $7.7549 \times 10^{3}$ \\
& $5 \times 10^{-3}$ & 12 & $7.7549 \times 10^{3}$ \\
& $5 \times 10^{-4}$ & Fail to converge with maximal iteration 100 \\
\hline & $5 \times 10^{0}$ & 59 & $7.7549 \times 10^{3}$ \\
& $5 \times 10^{-1}$ & 61 & $7.7549 \times 10^{3}$ \\
PCADM & $5 \times 10^{-2}$ & 60 & $7.7549 \times 10^{3}$ \\
& $5 \times 10^{-3}$ & 47 & $7.7549 \times 10^{3}$ \\
\hline \hline
\end{tabular}

\section{Acknowledgement}

The authors would like to thank the department of automation of southeast university, for providing the necessary facilities. This work is supported by State Grid Corporation of China, Major Projects on Planning and Operation Control of Large Scale Grid (SGCC-MPLG022-2012).

\section{References}

[1] Shoults, R. R., Chang, S. K., Helmick, S., \& Grady, W. M. (1980). A practical approach to unit commitment, economic dispatch and savings allocation for multiple-area pool operation with import/export constraints. IEEE Transactions on Power Apparatus and Systems, 99(2), 625-635.

[2] Yan, W., Wen, L., Li, W., Chung, C. Y., \& Wong, K. P. (2011). Decomposition-coordination interior point method and its application to multi-area optimal reactive power flow. International Journal of Electrical Power \& Energy Systems, 33(1), 55-60.

[3] Somasundaram, P., \& Swaroopan, N. M. J. (2011). Fuzzified particle swarm optimization algorithm for multi-area security constrained economic dispatch. Electric Power Components and Systems, 39(10), 979-990.

[4] Chung, K. H., Kim, B. H., \& Hur, D. (2011). Multi-area generation scheduling algorithm with regionally distributed optimal power flow using alternating direction method. International Journal of Electrical Power and Energy Systems, 33(9), 1527-1535.

[5] Basu, M. (2013). Artificial bee colony optimization for multi-area economic Dispatch. International Journal of Electrical Power and Energy Systems, 49, 181-187.

[6] Losi, A. (2003). On the application of the auxiliary problem principle. Journal of Optimization Theory and Applications, 117(2), 377-396. 
[7] He, B., \& Yuan, X. (2012). Convergence analysis of primal-dual algorithms for a saddle-point problem: From contraction perspective. SIAM Journal on Imaging Sciences, 5(1), 119-149.

[8] He, B. S., Tao, M., \& Yuan, X. M. (2012). Alternating direction method with gaussian back substitution for separable convex programming. SIAM Journal on Optimization, 22(2), 313-340.

[9] Chen, C. H., He, B. S., \& Yuan, X. M. (2012). Matrix completion via an alternating direction method. IMA Journal of Numerical Analysis, 32(1), 227-245.

[10] He, B. S., \& Sheng, Y. (2012). On the convergence rate of customized proximal point algorithm for convex optimization and saddle-point problem (in Chinese). SCI Sin Math, 42(5), 515-525.

[11] He, B., \& Hai, Y. (1998). Some convergence properties of a method of multipliers for linearly constrained monotone variational inequalities. Operations Research Letters, 23(3), 151-161.

[12] He, B. S., Yang, H., \& Wang, S. L. (2000). Alternating direction method with self-adaptive penalty parameters for monotone variational inequalities. Journal of Optimization Theory and Applications, 106(2), 337-356.

[13] Chen, P. H., \& Chang, H. C. (1995). Large-scale economic-dispatch by genetic algorithm. IEEE Transactions on Power Systems, 10(4), 1919-1926.

[14] Cohen, G. (1980). Auxiliary problem principle and decomposition of optimization problems. Journal of Optimization Theory and Applications, 32(3), 277-305.

[15] Kim, B. H., \& Baldick, R. (1997). Coarse-grained distributed optimal power flow. IEEE Transactions on Power Systems, 12(2), 932-939.

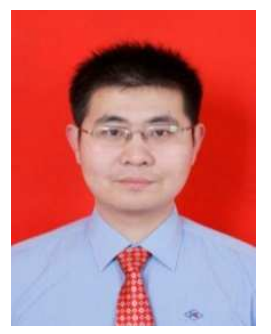

Yaming Ren was born in China in 1981, and received the bachelor degree and the master degree from the School of Electrical Engineering and Automation, Hefei University of Technology, Hefei, Anhui, China, in 2006 and 2009, respectively. Currently, he is a Ph.D. candidate in the Department of automation, Southeast University, Nanjing, Jiangsu, China. His research interests include power system planning and operation.

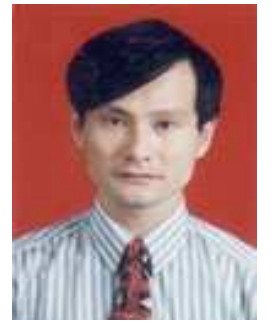

Shumin Fei was born in China in 1961, and received the Ph.D. degree from Beijing University of Aeronautics and Astronautics, China, in 1995. From 1995 to 1997, he was doing the postdoctoral research at the Research Institute of Automation, Southeast University, China. Presently, he is a professor and doctor adviser at Southeast University in China. He has published more than 70 journal papers and his current research interests include nonlinear systems, time-delay system, complex systems, and so on.

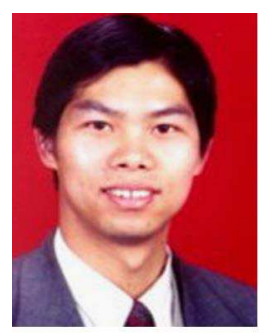

Haikun Wei was born in China in 1971, and received the B.S. degree from the Department of Automation, North China University of Technology, China in 1994, and the M.S. and Ph.D. degrees from the Research Institute of Automation, Southeast University, China in 1997 and 2000, respectively. He was a visiting scholar in RIKEN Brain Science Institute, Japan from 2005 to 2007. His research interests include real and artificial in neural networks and industry automation. 